\title{
The Role of High-Resolution Intracranial Vessel Wall Imaging in Intracranial Vasculopathies
}

Laisson de Moura Feitoza, MD

Fabiano Reis, MD, PhD

University of Campinas, Sao Paulo, Brazil 


\section{Purpose}

- To illustrate the spectrum of different intracranial vasculopathies using HR-VWI sequences

- To demonstrate the applicability of this technique in the differential diagnosis and aiding in detecting vascular involvement. 


\section{Background}

- Conventional imaging techniques for intracranial arteries (CTA, MRA and DSA) demonstrate abnormalities of the vessel lumen, but may fail to demonstrate vessel wall changes. The high-resolution vessel wall magnetic resonance imaging (HR-VWI) had been used due to its direct visualization of the vessel wall.

- In this exhibit we provided a pictorial review of some vasculopathies in which the technique may be useful in clinical practice. 
Pictorial review of intracranial vasculopathies that show the potential benefits of HR-VWI 

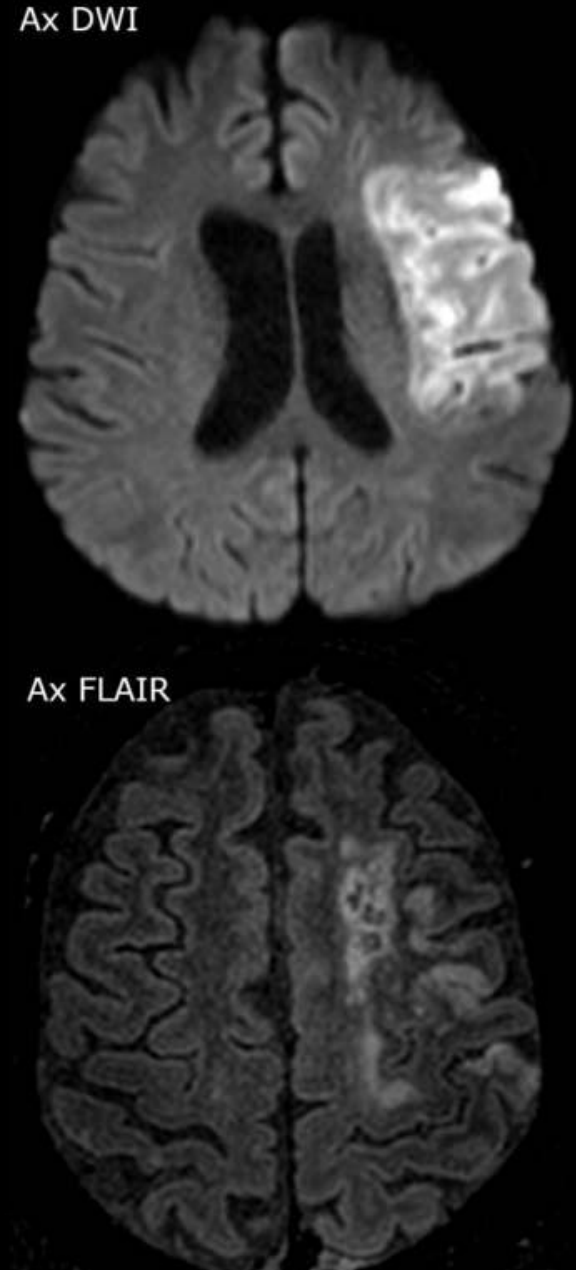

Ax DWI

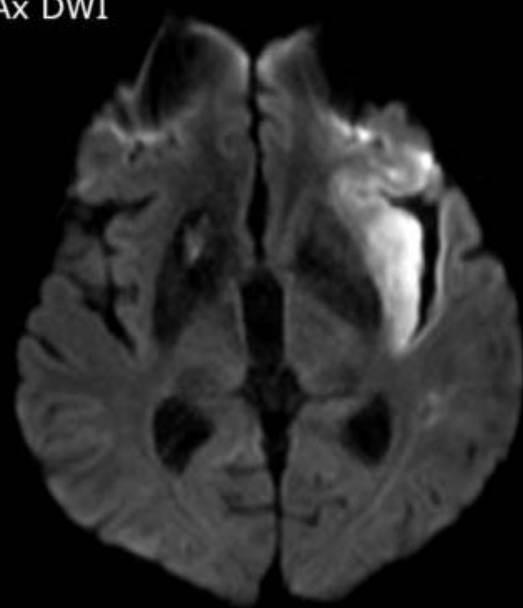

Ax FLAIR

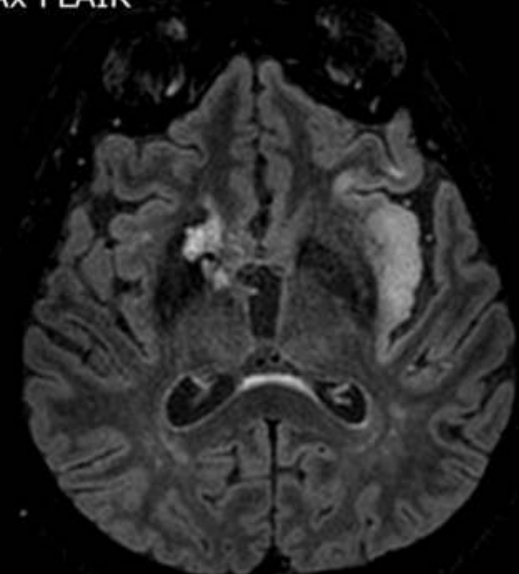

AX MRA
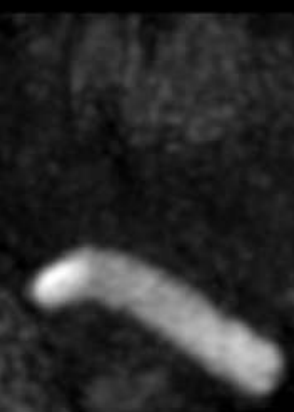

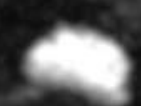

Ax HR-VWI

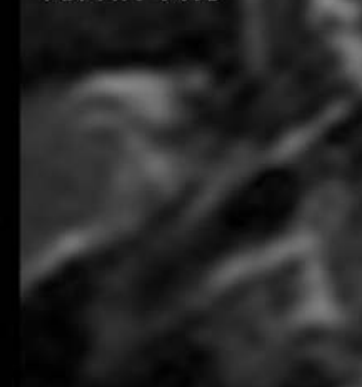

Giant cell arteritis. 69-year-old woman with giant cell arteritis admitted with seizures (Figure 2). 

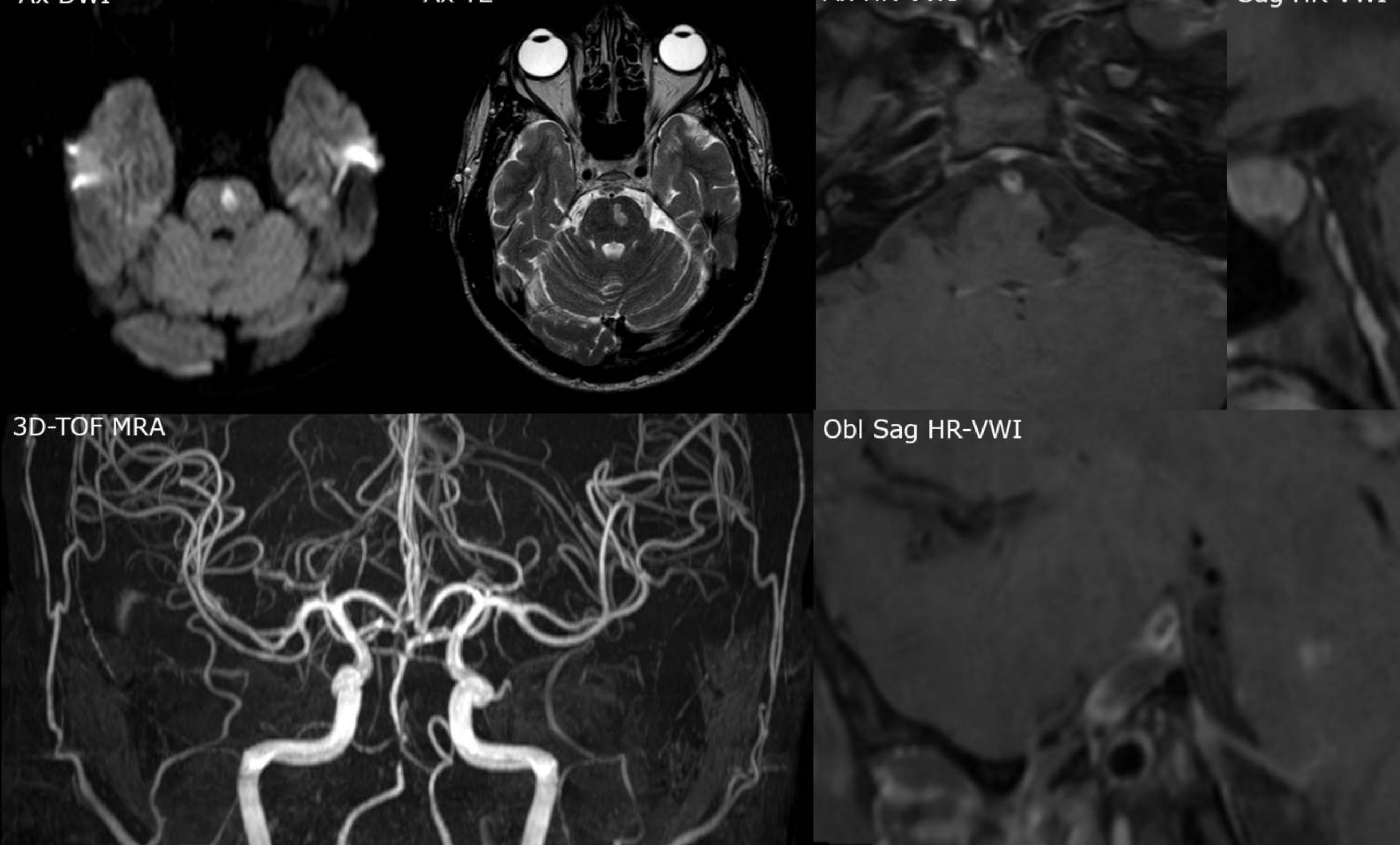

Obl Sag HR-VWI

Syphilis-associated vasculitis. A 26-year-old man presented acute-onset right hemiparesis, diplopia upon horizontal gaze and fever. The CSF and blood samples were positive for syphilis. A test for HIV was positive (Figure 4). 


\section{Conclusion}

- HR-VWI somehow transcribes the pathophysiological mechanism of vascular disease.

- It can be useful in cases where direct visualization of the vessel wall helps narrow the differential diagnosis (including infectious vasculitis) according to the pattern of involvement.

- Monitoring vasculitis treatment is also a potential benefit.

- This sequence also allowed to detect cranial nerve involvement (not detected in conventional sequences in this case). 


\section{Limitations}

- Further studies are needed to evaluate the accuracy of the technique. 